\title{
HARMONIC CONJUGATES OF WEIGHTED HARMONIC BERGMAN FUNCTIONS ON HALF-SPACES
}

\author{
Kyesook Nam and HeungSu Yi
}

\begin{abstract}
On the setting of the upper half-space of the Euclidean space $\mathbf{R}^{n}$, we show that to each weighted harmonic Bergman function $u \in b_{\alpha}^{p}$, there corresponds a unique conjugate system $\left(v_{1}, \ldots\right.$, $v_{n-1}$ ) of $u$ satisfying $v_{j} \in b_{\alpha}^{p}$ with an appropriate norm bound.
\end{abstract}

\section{Introduction}

For a fixed positive integer $n$, let $\mathbf{H}=\mathbf{R}^{n-1} \times \mathbf{R}_{+}$be the upper halfspace where $\mathbf{R}_{+}$denotes the set of all positive real numbers. We write point $z \in \mathbf{H}$ as $z=\left(z^{\prime}, z_{n}\right)$ where $z^{\prime} \in \mathbf{R}^{n-1}$ and $z_{n}>0$.

For $\alpha>-1$ and $1 \leq p<\infty$, let $b_{\alpha}^{p}(\mathbf{H})$ denote the weighted harmonic Bergman space consisting of all real-valued harmonic functions $u$ on $\mathbf{H}$ such that

$$
\|u\|_{L_{\alpha}^{p}}:=\left(\int_{\mathbf{H}}|u(z)|^{p} d V_{\alpha}(z)\right)^{1 / p}<\infty
$$

where $d V_{\alpha}(z)=z_{n}^{\alpha} d z$ and $d z$ is the Lebesgue measure on $\mathbf{R}^{n}$. We let $b_{\alpha}^{p}=b_{\alpha}^{p}(\mathbf{H})$ and $b^{p}=b_{0}^{p}$. Then we can check easily that the space $b_{\alpha}^{p}$ is a Banach space with the usual weighted $L^{p}$-norm.

Because $\mathbf{H}$ is a unbounded domain, it causes some problems. For example, the weighted harmonic Bergman kernel is not even integrable with respect to $d V_{\alpha}$ unlike the case of bounded domains. Fortunately,

Received September 3, 2002.

2000 Mathematics Subject Classification: Primary 31B05; Secondary 31B10, 30D45, 30D55.

Key words and phrases: harmonic Bergman function, harmonic conjugates, weighted Bergman kernel, fractional derivative, upper half-space.

This research was partially supported by KOSEF 2000-1-10100-001-3 and Kwangwoon University during sabbatical year in 2002 . 
not all the news about $\mathbf{H}$ is bad. One of the "nice" fact about $\mathbf{H}$ is that it is invariant under dilations, i.e., for every $r>0$,

$$
\{r z \mid z \in \mathbf{H}\}=\mathbf{H} \text {. }
$$

(There is nothing on the ball analogous to the dilation structure of $\mathbf{H}$.) This dilation invariant property gives us to use change of variable (especially with respect to the last coordinate) which helps us to estimate the size of some integrals that appear in the course of proofs.

Given a harmonic function $u$ on $\mathbf{H}$, the functions $v_{1}, \ldots, v_{n-1}$ on $\mathbf{H}$ are called harmonic conjugates of $u$ if

$$
\left(v_{1}, \cdots, v_{n-1}, u\right)=\nabla f
$$

for some function $f$ harmonic on $\mathbf{H}$, where $\nabla f$ denotes the gradient of $f$. In this case we call $\left(v_{1}, \ldots, v_{n-1}\right)$ a conjugate system of $u$. Note that if (1.1) holds, then $v_{1}, \ldots, v_{n-1}$ are partial derivatives of a harmonic function, so they are harmonic on $\mathbf{H}$. Also, (1.1) and the condition that $f$ is harmonic are equivalent to the following "generalized CauchyRiemann equations": for $j, k=1, \ldots, n-1$,

$$
\begin{gathered}
D_{k} v_{j}=D_{j} v_{k} ; D_{n} v_{j}=D_{j} u, \\
\sum_{j=1}^{n-1} D_{j} v_{j}+D_{n} u=0 .
\end{gathered}
$$

It is well known that a conjugate system of a given harmonic function always exists on $\mathbf{H}$. Unfortunately, a conjugate system is far from unique. In fact if $n>2$, then harmonic conjugates of a given function $u$ may well differ by more than a constant. We refer more on harmonic conjugates to [4] and [5].

It is shown in [3] that for a given function $u \in b^{p}$, there exists a unique conjugate system $\left(v_{1}, \ldots, v_{n-1}\right)$ of $u$ satisfying $v_{j} \in b^{p}$ for each $j$.

In this paper as stated below, we show that this result also holds for weighted harmonic Bergman spaces $b_{\alpha}^{p}$ for any range $\alpha>-1$.

THEOREM 1. Let $\alpha>-1$ and let $1 \leq p<\infty$. Then to each $u \in b_{\alpha}^{p}$, there corresponds a unique conjugate system $\left(v_{1}, \ldots, v_{n-1}\right)$ of $u$ such that $v_{j} \in b_{\alpha}^{p}$. Moreover,

$$
\sum_{j=1}^{n-1}\left\|v_{j}\right\|_{L_{\alpha}^{p}} \approx\|u\|_{L_{\alpha}^{p}}
$$

as $u$ ranges over all functions in $b_{\alpha}^{p}$. 
This paper is organized as follows. In section 2, we review some results about the extended Poisson kernel and its fractional derivatives on $\mathbf{H}$. Also, we show some lemmas to prove Theorem 1. Section 3 is devoted to the proof of Theorem 1 .

Constants. Throughout the paper we use the same letter $C$ to denote various constants which may change at each occurrence. The constant $C$ may often depend on the dimension $n$ and some other parameters, but it is always independent of particular functions, points or parameters under consideration. For nonnegative quantities $A$ and $B$, we often write $A \lesssim B$ if $A$ is dominated by $B$ times some inessential positive constant. Also, we write $A \approx B$ if $A \lesssim B$ and $B \lesssim A$.

\section{Preliminary results}

In this section, we review some preliminary results about the weighted harmonic Bergman kernel and its related facts. Because its explicit form takes the fractional derivative of the extended Poisson kernel, we start with the extended Poisson kernel on the upper half-space.

Let $P(z, w)$ be the extended Poisson kernel on $\mathbf{H}$, i.e.,

$$
P_{z}(w):=P(z, w)=\frac{2}{n V(B)} \frac{z_{n}+w_{n}}{|z-\bar{w}|^{n}}
$$

where $z, w \in \mathbf{H}$ and $\bar{w}=\left(w^{\prime},-w_{n}\right)$. Note that for each $j=1, \ldots, n-1$, $D_{z_{j}} P(z, w)=-D_{w_{j}} P(z, w)$ and $D_{z_{n}} P(z, w)=D_{w_{n}} P(z, w)$. Therefore we can show from $(2.1)$ that for multi-indices $\mathbf{m}=\left(m_{1}, \ldots, m_{n}\right)$ and $\mathbf{k}=\left(k_{1}, \ldots, k_{n}\right)$,

$$
\begin{aligned}
D_{z}^{\mathbf{m}} D_{w}^{\mathbf{k}} P(z, w) & =D_{z_{1}}^{m_{1}} \cdots D_{z_{n}}^{m_{n}} D_{w_{1}}^{k_{1}} \cdots D_{w_{n}}^{k_{n}} P(z, w) \\
& =(-1)^{k_{1}+\cdots+k_{n-1}} D_{z_{1}}^{m_{1}+k_{1}} \cdots D_{z_{n}}^{m_{n}+k_{n}} P(z, w) \\
& =(-1)^{k_{1}+\cdots+k_{n-1}} \frac{f_{\mathbf{m}, \mathbf{k}}(z-\bar{w})}{|z-\bar{w}|^{n+2|\mathbf{m}|+2|\mathbf{k}|}}
\end{aligned}
$$

where $f_{\mathbf{m}, \mathbf{k}}$ is a homogeneous polynomial of degree $1+|\mathbf{m}|+|\mathbf{k}|$. Thus,

$$
\left|D_{z}^{\mathbf{m}} D_{w}^{\mathbf{k}} P(z, w)\right| \lesssim \frac{1}{|z-\bar{w}|^{n+|\mathbf{m}|+|\mathbf{k}|-1}} .
$$

Let $D=D_{n}$ denote the differentiation with respect to the last component. Then we define the fractional derivative of the extended Poisson kernel of order $s$ for $s \geq 0$ as follows: If $s$ is a nonnegative integer, then

$$
\mathcal{D}^{s} P_{z}(w)=D^{s} P_{z}(w) \text {. }
$$


If $s$ is not a nonnegative integer, then

$$
\mathcal{D}^{s} P_{z}(w)=\frac{1}{\Gamma([s]-s)} \int_{0}^{\infty} t^{[s]-s-1} D^{[s]} P_{z}\left(w^{\prime}, w_{n}+t\right) d t
$$

where $\Gamma$ is a gamma function and $[s]$ is the smallest integer greater than or equal to $s$. In this case, we know from (2.2) that the above integral always makes sense, because $[s]-s-1>-1$ and $n+s>1$. We get the idea of this fractional derivative from [1].

Let $\beta>-1$ and let

$$
R_{\beta}(z, w)=\frac{1}{C_{\beta}} \mathcal{D}^{\beta+1} P_{z}(w)
$$

on $\mathbf{H} \times \mathbf{H}$, where

$$
C_{\beta}=\frac{(-1)^{[\beta]+1}([\beta]+1) !}{\Gamma([\beta]-\beta+1)} \int_{0}^{\infty} \frac{t^{[\beta]-\beta}}{(2+t)^{[\beta]+2}} d t .
$$

Then it is shown in [2] that $R_{\alpha}(z, w)$ is the weighted harmonic Bergman kernel for $b_{\alpha}^{2}$ and that for $\beta \geq \alpha, R_{\beta}(z, w)$ reproduces all functions in $b_{\alpha}^{p}$ for every range $1 \leq p<\infty$, i.e., for $1 \leq p<\infty, u \in b_{\alpha}^{p}, z \in \mathbf{H}$,

$$
u(z)=\int_{\mathbf{H}} u(w) R_{\beta}(z, w) d V_{\beta}(w)
$$

whenever $\beta \geq \alpha$. Furthermore it is also shown in [2] that for $\alpha>-1$, $1 \leq p<\infty$,

$$
\|u\|_{L_{\alpha}^{p}} \approx\left\|z_{n} D_{n} u\right\|_{L_{\alpha}^{p}}
$$

as $u$ ranges over all $b_{\alpha}^{p}$-functions. In other words, the weighted Bergman norm is equivalent to the weighted normal derivative norm. (See [2] for details and related facts.)

The following lemma is used to estimate the size of some integrals that appear in the course of proofs.

Lemma 2. For $b<0, a+b>-1$, we have

$$
\int_{\mathbf{H}} \frac{w_{n}^{a+b}}{|z-\bar{w}|^{n+a}} d w \lesssim z_{n}^{b}
$$

for every $z, w \in \mathbf{H}$. 
Proof. Using polar coordinates centered at $z^{\prime}$ on $\mathbf{R}^{n-1}$, we have

$$
\begin{aligned}
& \int_{\mathbf{H}} \frac{w_{n}^{a+b}}{|z-\bar{w}|^{n+a}} d w \\
= & \int_{0}^{\infty} \int_{\mathbf{R}^{n-1}} \frac{w_{n}^{a+b}}{\left(\left|z^{\prime}-w^{\prime}\right|^{2}+\left(z_{n}+w_{n}\right)^{2}\right)^{(n+a) / 2}} d w^{\prime} d w_{n} \\
\lesssim & \int_{0}^{\infty} \int_{0}^{\infty} \frac{r^{n-2} w_{n}^{a+b}}{\left(r+\left(z_{n}+w_{n}\right)\right)^{n+a}} d r d w_{n} \\
\lesssim & \int_{0}^{\infty} \int_{0}^{z_{n}+w_{n}} \frac{r^{n-2} w_{n}^{a+b}}{\left(z_{n}+w_{n}\right)^{n+a}} d r d w_{n}+\int_{0}^{\infty} \int_{z_{n}+w_{n}}^{\infty} \frac{w_{n}^{a+b}}{r^{a+2}} d r d w_{n} \\
\lesssim & \int_{0}^{\infty} \frac{w_{n}^{a+b}}{\left(z_{n}+w_{n}\right)^{a+1}} d w_{n} \\
\lesssim & \int_{0}^{z_{n}} \frac{w_{n}^{a+b}}{z_{n}^{a+1}} d w_{n}+\int_{z_{n}}^{\infty} w_{n}^{b-1} d w_{n} \lesssim z_{n}^{b} .
\end{aligned}
$$

The proof is complete.

Now we estimate the size of derivatives of $R_{\beta}(z, w)$.

LEMmA 3. Let $\beta>-1$. Then for multi-indices $\mathbf{m}$ and $\mathbf{k}$,

for $z, w \in \mathbf{H}$.

$$
\left|D_{z}^{\mathbf{m}} D_{w}^{\mathbf{k}} R_{\beta}(z, w)\right| \lesssim \frac{1}{|z-\bar{w}|^{n+\beta+|\mathbf{m}|+|\mathbf{k}|}}
$$

Proof. If $\beta$ is a nonnegative integer, then we get the desired result from (2.2). Assume that $\beta$ is not an integer. Then note from (2.2) that $\left|D_{z}^{\mathbf{m}} D_{w}^{\mathbf{k}} D^{[\beta]+1} P\left(z,\left(w^{\prime}, w_{n}+t\right)\right)\right| \lesssim\left|z-\left(w^{\prime},-\left(w_{n}+t\right)\right)\right|^{-(n+[\beta]+|\mathbf{m}|+|\mathbf{k}|)}$ and note also that

$$
\left|z-\left(w^{\prime},-\left(w_{n}+t\right)\right)\right| \approx|z-\bar{w}|+t
$$

for $z, w \in \mathbf{H}$ and for $t>0$. Thus,

$$
\begin{aligned}
\left|D_{z}^{\mathbf{m}} D_{w}^{\mathbf{k}} R_{\beta}(z, w)\right| & \lesssim \int_{0}^{\infty}\left|D_{z}^{\mathbf{m}} D_{w}^{\mathbf{k}} D^{[\beta]+1} P\left(z,\left(w^{\prime}, w_{n}+t\right)\right)\right| t^{[\beta]-\beta-1} d t \\
& \lesssim \int_{0}^{\infty} \frac{t^{[\beta]-\beta-1}}{(|z-\bar{w}|+t)^{n+[\beta]+|\mathbf{m}|+|\mathbf{k}|}} d t \\
& \approx \frac{1}{|z-\bar{w}|^{n+\beta+|\mathbf{m}|+|\mathbf{k}|}},
\end{aligned}
$$

where we used change of variable $t \mapsto|z-\bar{w}| t$. This completes the proof. 
Define $\Pi$ on the weighted Lebesgue space $L_{\alpha}^{p}$ by

$$
\Pi f(z)=\int_{\mathbf{H}} f(w) \frac{w_{n}^{\alpha+1}}{|z-\bar{w}|^{n+\alpha+1}} d w
$$

for each $f \in L_{\alpha}^{p}$ and for every $z \in \mathbf{H}$. Then we show in the following lemma $\Pi$ is bounded on $L_{\alpha}^{p}$.

Lemma 4. Let $\alpha>-1$ and let $1 \leq p<\infty$. Then $\Pi$ is bounded on $L_{\alpha}^{p}$.

Proof. If $p=1$, then we see from Lemma 2 that for $f \in L_{\alpha}^{1}$

$$
\begin{aligned}
\|\Pi f\|_{L_{\alpha}^{1}} & \leq \int_{\mathbf{H}}|f(w)| \int_{\mathbf{H}} \frac{z_{n}^{\alpha}}{|z-\bar{w}|^{n+\alpha+1}} d z w_{n}^{\alpha+1} d w \\
& \lesssim \int_{\mathbf{H}}|f(w)| w_{n}^{-1+\alpha+1} d w \\
& =\|f\|_{L_{\alpha}^{1}} .
\end{aligned}
$$

Let $1<p<\infty, f \in L_{\alpha}^{p}$ and let $q$ denote the index conjugate to $p$. Choose a real number $s$ satisfying both $-(1+\alpha) / p<s<0$ and $-(2+\alpha) / q<s$. Then after applying Hölder's inequality to the following two functions

$$
|f(w)|\left(\frac{w_{n}^{\alpha+1-p s}}{|z-\bar{w}|^{n+\alpha+1}}\right)^{1 / p}, \quad\left(\frac{w_{n}^{\alpha+1+q s}}{|z-\bar{w}|^{n+\alpha+1}}\right)^{1 / q}
$$

we see from Lemma 2 that

$$
\begin{aligned}
|\Pi f(z)| & \leq \int_{\mathbf{H}}|f(w)| \frac{w_{n}^{\alpha+1}}{|z-\bar{w}|^{n+\alpha+1}} d w . \\
& \lesssim\left(\int_{\mathbf{H}}|f(w)|^{p} \frac{w_{n}^{\alpha+1-s p}}{|z-\bar{w}|^{n+\alpha+1}} d w\right)^{1 / p}\left(\int_{\mathbf{H}} \frac{w_{n}^{\alpha+1+s q}}{|z-\bar{w}|^{n+\alpha+1}} d w\right)^{1 / q} \\
& \lesssim z_{n}^{s}\left(\int_{\mathbf{H}}|f(w)|^{p} \frac{w_{n}^{\alpha+1-s p}}{|z-\bar{w}|^{n+\alpha+1}} d w\right)^{1 / p},
\end{aligned}
$$

because $\alpha+1+s q>-1$ and $s q<0$. After applying Lemma 2 once again, we see that

$$
\begin{aligned}
\|\Pi f\|_{L_{\alpha}^{p}}^{p} & \lesssim \int_{\mathbf{H}}|f(w)|^{p} w_{n}^{\alpha+1-s p} \int_{\mathbf{H}} \frac{z_{n}^{\alpha+s p}}{|z-\bar{w}|^{n+\alpha+1}} d z d w \\
& \lesssim\|f\|_{L_{\alpha}^{p}}^{p}
\end{aligned}
$$

because $\alpha+s p>-1$ and $s p-1<0$. Therefore the proof is complete.

Before we prove the main theorem, we show one more lemma. 
LEMmA 5. Let $\alpha>-1,1 \leq p<\infty$ and let $u \in b_{\alpha}^{p}(\mathbf{H})$. Then for each $j=1, \ldots, n$,

$$
\left\|z_{n} D_{j} u\right\|_{L_{\alpha}^{p}} \lesssim\|u\|_{L_{\alpha}^{p}}
$$

Proof. We know from (2.4) with $\beta=\alpha+1$ that

$$
u(z)=\int_{\mathbf{H}} u(w) R_{\alpha+1}(z, w) w_{n}^{\alpha+1} d w
$$

for $z \in \mathbf{H}$. Differentiating through the integral above, we see that for each $j$,

$$
z_{n} D_{j} u(z)=\int_{\mathbf{H}} u(w) z_{n}\left[D_{z_{j}} R_{\alpha+1}(z, w)\right] w_{n}^{\alpha+1} d w .
$$

Notice from Lemma 3 that

$$
\left|z_{n} D_{z_{j}} R_{\alpha+1}(z, w)\right| \lesssim \frac{z_{n}}{|z-\bar{w}|^{n+\alpha+2}} \leq \frac{1}{|z-\bar{w}|^{n+\alpha+1}} .
$$

Thus for each $j$

$$
\left|z_{n} D_{j} u(z)\right| \lesssim \int_{\mathbf{H}}|u(w)| \frac{w_{n}^{\alpha+1}}{|z-\bar{w}|^{n+\alpha+1}} d w .
$$

Therefore the desired result follows directly from Lemma 4 and the proof is complete.

\section{Proof of Theorem 1}

For each $j=1, \ldots, n-1$ and for every $z \in \mathbf{H}$, set

$$
v_{j}(z)=-\frac{C_{\alpha}}{C_{\alpha+1}} \int_{\mathbf{H}} u(w) D_{w_{j}} R_{\alpha}(z, w) w_{n}^{\alpha+1} d w,
$$

where $C_{\alpha}$ is a constant given in (2.3). Then by passing the Laplacian through the integral above, we see that each $v_{j}$ is harmonic on $\mathbf{H}$. Assume that $\alpha$ is not an integer. Note that for each $j$ and for every $z, w \in \mathbf{H}$,

$$
\begin{aligned}
D_{w_{j}} R_{\alpha}(z, w) & =\frac{1}{C_{\alpha}} \int_{0}^{\infty}\left[D_{w_{j}} D_{w_{n}}^{[\alpha]+1} P_{z}\left(w^{\prime}, w_{n}+t\right)\right] t^{[\alpha]-\alpha-1} d t \\
& =-\frac{1}{C_{\alpha}} \int_{0}^{\infty}\left[D_{z_{j}} D_{w_{n}}^{[\alpha]+1} P_{z}\left(w^{\prime}, w_{n}+t\right)\right] t^{[\alpha]-\alpha-1} d t \\
& =-D_{z_{j}} R_{\alpha}(z, w) .
\end{aligned}
$$


Fix $z \in \mathbf{H}$. Then for each $1 \leq j, k \leq n-1$,

$$
\begin{aligned}
D_{k} v_{j}(z) & =-\frac{C_{\alpha}}{C_{\alpha+1}} \int_{\mathbf{H}} u(w)\left[D_{z_{k}} D_{w_{j}} R_{\alpha}(z, w)\right] w_{n}^{\alpha+1} d w \\
& =-\frac{C_{\alpha}}{C_{\alpha+1}} \int_{\mathbf{H}} u(w)\left[D_{z_{j}} D_{w_{k}} R_{\alpha}(z, w)\right] w_{n}^{\alpha+1} d w \\
& =D_{j} v_{k}(z) .
\end{aligned}
$$

Because $[\alpha]-\alpha=[\alpha+1]-(\alpha+1)$,

$$
C_{\alpha} D_{w_{n}} R_{\alpha}(z, w)=C_{\alpha+1} R_{\alpha+1}(z, w) .
$$

Therefore we see from (2.4) that for each $j$,

$$
\begin{aligned}
D_{n} v_{j}(z) & =-\frac{C_{\alpha}}{C_{\alpha+1}} \int_{\mathbf{H}} u(w)\left[D_{z_{n}} D_{w_{j}} R_{\alpha}(z, w)\right] w_{n}^{\alpha+1} d w \\
& =\int_{\mathbf{H}} u(w)\left[D_{z_{j}} R_{\alpha+1}(z, w)\right] w_{n}^{\alpha+1} d w \\
& =D_{j} u(z) .
\end{aligned}
$$

Clearly,

$$
\sum_{j=1}^{n-1} D_{j} v_{j}(z)+D_{n} u(z)=\frac{C_{\alpha}}{C_{\alpha+1}} \int_{\mathbf{H}} u(w) \Delta_{z} R_{\alpha}(z, w) w_{n}^{\alpha+1} d w=0 .
$$

This shows that $v_{1}, \ldots, v_{n-1}, u$ satisfy the generalized Cauchy-Riemann equations. Thus $\left(v_{1}, \ldots, v_{n-1}\right)$ is a conjugate system of $u$.

Note that

$$
\left|v_{j}(z)\right| \lesssim \int_{\mathbf{H}}|u(w)| \frac{w_{n}^{\alpha+1}}{|z-\bar{w}|^{n+\alpha+1}} d w .
$$

Then we get from Lemma 4 that $\left\|v_{j}\right\|_{L_{\alpha}^{p}} \lesssim\|u\|_{L_{\alpha}^{p}}$ for each $j$. Hence,

$$
\sum\left\|v_{j}\right\|_{L_{\alpha}^{p}} \lesssim\|u\|_{L_{\alpha}^{p}}
$$

For the other inequality, we get from (2.5) and (3.1) that

$$
\|u\|_{L_{\alpha}^{p}} \approx\left\|z_{n} D_{n} u\right\|_{L_{\alpha}^{p}}=\left\|z_{n}\left(-\sum D_{j} v_{j}\right)\right\|_{L_{\alpha}^{p}} \leq \sum\left\|z_{n} D_{j} v_{j}\right\|_{L_{\alpha}^{p} .}
$$

Because $v_{j} \in b_{\alpha}^{p}$, we see from Lemma 5 that for each $j$,

$$
\left\|z_{n} D_{j} v_{j}\right\|_{L_{\alpha}^{p}} \lesssim\left\|v_{j}\right\|_{L_{\alpha}^{p}}
$$

Therefore we get

$$
\|u\|_{L_{\alpha}^{p}} \lesssim \sum\left\|v_{j}\right\|_{L_{\alpha}^{p}}
$$


Consequently, we have

$$
\|u\|_{L_{\alpha}^{p}} \approx \sum_{j=1}^{n-1}\left\|v_{j}\right\|_{L_{\alpha}^{p}} .
$$

For the uniqueness, suppose that $\left(u_{1}, \ldots, u_{n-1}\right)$ is also a conjugate system of $u$ satisfying $u_{j} \in b_{\alpha}^{p}(\mathbf{H})$ for each $j$. Then by (2.5), we obtain

$$
\left\|v_{j}-u_{j}\right\|_{L_{\alpha}^{p}} \approx\left\|z_{n} D_{n}\left(v_{j}-u_{j}\right)\right\|_{L_{\alpha}^{p}}=\left\|z_{n} D_{j}(u-u)\right\|_{L_{\alpha}^{p}}=0 .
$$

Thus, $v_{j}=u_{j}$ for each $j$ and this completes the proof of the case that $\alpha$ is not an integer.

If $\alpha$ is an integer, then we get the desired result, similarly (much easier than the previous case). Therefore the proof is complete.

\section{References}

[1] F. Beatrous, Behavior of holomorphic functions near weakly pseudoconvex boundary points, Indiana Univ. Math. J. 40 (1991), no. 3, 915-966.

[2] H. Koo, K. S. Nam and H. Yi, Weighted harmonic Bergman kernel and its applications on half-spaces, Preprint.

[3] W. Ramey and H. Yi, Harmonic Bergman functions on half-spaces, Trans. Amer. Math. Soc. 348 (1996), 633-660.

[4] E. Stein, Singular integrals and differentiablity of functions, Princeton University Press, Princeton, 1970.

[5] E. Stein and G. Weiss, Fourier analysis on euclidean spaces, Princeton University Press, Princeton, 1971.

Department of Mathematics

Korea University

Seoul 136-701, Korea

E-mail: ksnam@semi.korea.ac.kr

Department of Mathematics

Kwangwoon University

Seoul 139-701, Korea

E-mail: hsyi@kwangwoon.ac.kr 